\title{
Review Article \\ Urinary Markers of Tubular Injury in Early Diabetic Nephropathy
}

\author{
Temesgen Fiseha and Zemenu Tamir \\ Department of Clinical Laboratory Science, College of Medicine and Health Sciences, Wollo University, Dessie, Ethiopia \\ Correspondence should be addressed to Temesgen Fiseha; temafiseha@gmail.com
}

Received 22 February 2016; Accepted 26 April 2016

Academic Editor: Franca Anglani

Copyright ( 92016 T. Fiseha and Z. Tamir. This is an open access article distributed under the Creative Commons Attribution License, which permits unrestricted use, distribution, and reproduction in any medium, provided the original work is properly cited.

Diabetic nephropathy (DN) is a common and serious complication of diabetes associated with adverse outcomes of renal failure, cardiovascular disease, and premature mortality. Early and accurate identification of DN is therefore of critical importance to improve patient outcomes. Albuminuria, a marker of glomerular involvement in early renal damage, cannot always detect early DN. Thus, more sensitive and specific markers in addition to albuminuria are needed to predict the early onset and progression of DN. Tubular injury, as shown by the detection of tubular injury markers in the urine, is a critical component of the early course of DN. These urinary tubular markers may increase in diabetic patients, even before diagnosis of microalbuminuria representing early markers of normoalbuminuric DN. In this review we summarized some new and important urinary markers of tubular injury, such as neutrophil gelatinase associated lipocalin (NGAL), kidney injury molecule-1 (KIM-1), liver-type fatty acid binding protein (L-FABP), N-acetyl-beta-glucosaminidase (NAG), alpha-1 microglobulin (A1M), beta 2-microglobulin (B2-M), and retinol binding protein (RBP) associated with early DN.

\section{Introduction}

Diabetic nephropathy (DN) is a common and serious complication of diabetes associated with adverse outcomes of renal failure, cardiovascular disease, and premature mortality [1-3]. It is also the leading cause of end-stage renal disease (ESRD), requiring costly renal replacement therapy in the form of dialysis or transplantation [4]. Early and accurate identification of DN is therefore of critical importance to improve clinical outcomes. Clinically, the appearance of pathological albuminuria, microalbuminuria, is considered a hallmark of early onset of DN. However, a substantial proportion of renal impairment occurs among normoalbuminuric diabetic patients and is associated with more advanced diabetic glomerular lesions and increased risk of progression [5-7]. Moreover, since microalbuminuria is diagnosed once significant glomerular damage has occurred, changes in albuminuria are being increasingly recognized as complementary rather than obligatory manifestations of DN $[8,9]$.

Recently, changes in the renal tubules, which may be termed diabetic tubulopathy, are increasingly implicated in the development of progressive diabetic kidney disease [10, 11]. It has been reported that, in addition to the glomeruli, the renal tubules are heavily involved in the pathogenesis of DN [12, 13]. In line with this, several glomerular and tubular biomarkers predicting onset or progression of $\mathrm{DN}$ have been identified and are becoming increasingly important in clinical diagnostics. The urinary concentrations of these damage markers (both glomerular and tubular) are elevated in diabetic patients and are associated with the severity of DN [14]. Interestingly, some of these markers already are elevated in normoalbuminuric diabetic patients with normal estimated glomerular filtration rate (eGFR) [14]. Therefore, more sensitive and specific markers in addition to albuminuria are needed to predict the development and progression of $\mathrm{DN}$ in diabetics even at the very early stage (normoalbuminuric DN).

Tubular injury, as shown by the detection of tubular damage markers in the urine, is a critical component of the early course of $\mathrm{DN}$ and has been suggested to contribute in a primary way, rather than a secondary manner, to the development of early DN $[15,16]$. Urinary excretion of these 
tubular markers could, therefore, be useful for assessing an initial malfunction or damage of the renal tubules in the early stage of potentially progressive DN. Several tubular damage markers recently have been discovered and have clinical implications as markers for the development and progression of DN. Some studies have demonstrated that these tubular damage markers increase in patients with diabetes, even before diagnosis of microalbuminuria representing early markers of normoalbuminuric $\mathrm{DN}$ with a good sensitivity and specificity [17-19]. This review article summarizes some new and important urinary markers of tubular injury associated with early DN.

\section{Neutrophil Gelatinase Associated Lipocalin}

Neutrophil gelatinase associated lipocalin (NGAL) is a small, $25-\mathrm{kDa}$, protein that belongs to the lipocalin protein family released from neutrophils and many epithelial cell types including kidney tubular cells. It is representative of the functioning tubular mass and produced as a response to tubular injury [20]. Urinary NGAL levels were found to be markedly elevated in patients with diabetes when compared with nondiabetic control subjects [14, 21-27] and to correlate negatively with eGFR and positively with serum Cystatin $\mathrm{C}(\mathrm{Cys})$, serum creatinine (SCr), proteinuria, albuminuria, and urinary albumin excretion (UAE) and albumincreatinine ratio (UACR), indicating the possible clinical application of urinary NGAL as a complementary marker for early detection of DN [19, 25-29]. It is also significantly correlated with the duration of diabetes, glycemic control (HbAlc), and urinary interleukin-18 (IL-18: proinflammatory biomarker) and angiotensinogen (renin-angiotensin system biomarker), suggesting urinary NGAL as a useful noninvasive tool for the evaluation of renal involvement in diabetes $[21,23,25,28,29]$.

In diabetics, urinary excretion of NGAL was significantly higher in microalbuminuric in comparison with normoalbuminuric patients and controls and correlated positively with UACR, indicating diabetic tubular damage at the early stage of DN $[18,23,25,26,30]$. Urine levels of NGAL were significantly higher in microalbuminuria group compared to normoalbuminuria and were positively correlated to UACR in both diabetes and prediabetes, which suggested that tubular damage may play major role in the development of nephropathy in prediabetes [30]. It was also suggested that NGAL might play an important role in the pathophysiology of renal adaptation to diabetes, and its measurement might become a useful and noninvasive tool for the evaluation of renal involvement in these patients as well as for the early diagnosis of incipient DN [26]. In addition, urinary NGAL showed better area under curve (AUC; diagnostic accuracy) for estimating microalbuminuria, demonstrating its value as a more suitable and sensitive marker for detecting the onset of DN $[25,30]$.

Higher urinary levels of NGAL have been found in diabetics compared to controls, even in normoalbuminuric patients, indicating that diabetic tubular damage may develop before the stage of microalbuminuria [14, 17, 22, 23, 26, 31, 32]. The urinary NGAL level (1.5-fold) already was significantly elevated in normoalbuminuric patients with diabetes compared with nondiabetic controls and was significantly associated with albuminuria [14]. Mean urinary NGAL and NGAL/creatinine ratio (NGAL/Cr) levels in both microalbuminuric and nonmicroalbuminuric diabetic patients were found to be higher than those in the controls, indicating that tubular involvement may precede glomerular involvement, as urinary NGAL levels are increased in the very early phase of diabetes before microalbuminuria develops [24].

The increased levels of urinary NGAL in diabetic patients, with or without albuminuria, point to early tubular damage and can be used as an early sensitive marker in detecting DN [14, 22, 27, 31]. In addition, urinary NGAL levels were increased in diabetic patients with normal or mildly increased albuminuria, which indicated that tubular and glomerular injuries may be occurring even at the earliest stage of diabetic kidney disease and urinary NGAL could be an early marker of renal dysfunction in diabetic patients without current evidence of nephropathy $[19,31]$.

NGAL increases in diabetic patients, even before diagnosis of microalbuminuria representing an early marker of "normoalbuminuric" DN, and could be used for the evaluation of early renal involvement in the course of diabetes [17]. Urine NGAL was significantly increased in diabetic patients, even normoalbuminuric, and was positively correlated with $\mathrm{HbAlc}$, duration of diabetes, and urine ACR, suggesting that urinary NGAL could have the potential to be an earlier marker of $\mathrm{DN}$, in normoalbuminuric patients, as a supplement to albuminuria [23]. In addition, urinary levels of NGAL were higher in normoalbuminuric patients with diabetes than in control subjects and increased with increasing categories of albuminuria [14, 29].

Because levels of urinary NGAL were significantly different according to the degree of albuminuria and increased in parallel with the severity of renal disease, reaching higher levels in patients with manifest DN, urinary NGAL levels expresses the degree of renal impairment in DN and, together with albuminuria, it could be used as a sensitive and specific markers for predicting the progression of DN $[14,17,19,22,26,31]$. Furthermore, baseline levels of urinary NGAL were significantly elevated and correlated with the severity of albuminuria in patients with diabetes and were observed to be significantly correlated with a rapid decline in the eGFR [33]. Elevated levels of urinary NGAL are also predictive of decline in eGFR in type 2 diabetic patients with micro- or macroalbuminuria [34]. Another study also found that urinary concentrations of NGAL were associated with progression to ESRD and death in type 2 diabetes, even after adjustment for baseline albuminuria and GFR, indicating the potential importance of urinary NGAL for the identification of persons most likely to progress to ESRD or to premature death [35].

\section{Kidney Injury Molecule-1}

Kidney injury molecule-1 (KIM-1) is a transmembrane protein and its expression is not measurable in normal proximal tubule cells but is markedly upregulated with injury/dedifferentiation [36]. It has been suggested that its presence in 
the urine is highly specific for kidney injury and may serve as a useful biomarker for renal proximal tubule injury facilitating the early diagnosis of the disease [36, 37]. Renal tubular damage, as evidenced by increased levels of urinary KIM-1, is evident even prior to the development of diabetes and overt kidney disease [38].

Urinary levels of KIM-1 were significantly higher in patients with diabetes when compared with nondiabetic control subjects and correlated with urinary albumin, UACR, $\mathrm{SCr}$, blood urea, and BUN, indicating the possible clinical application of urinary KIM-1 as a complementary marker for early detection of DN $[14,18,21,22,27,39]$. Urinary KIM-1 levels have also been found to be correlated with the urinary IL-18 and angiotensinogen, body mass index, duration of diabetes, glycemic control, and systolic and diastolic blood pressures (BP), which may reflect the role of Kim-1 as a marker for diagnosis and prognosis of $\mathrm{DN}$ among diabetic patients taking into account other risk factors [19, 21, 39-41]. In addition, urinary KIM-1 levels were significantly increased both in subgroups of DN and in chronic kidney disease (CKD) compared with controls [41]. In this study, urinary KIM-1 levels, along with urinary albumin excretion and the duration of diabetes, were found to be independent risk factors associated with low GFRs [41].

Urinary levels of KIM-1 were found significantly elevated in diabetic patients with microalbuminuria, in comparison with diabetics with normoalbuminuria and nondiabetic healthy controls, demonstrating the existence of diabetic tubular damage at the early stage of DN $[16,18,21,39,41]$. In one study, urinary Kim-1 levels were elevated significantly (10-fold) in type 2 diabetic microalbuminuric patients (whose SCr level was $<2 \mathrm{mg} / \mathrm{dL}$ ) as compared to the controls and normoalbuminuric patients, indicating its potential value in the identification of diabetics with nephropathy at the early stage [39]. Urinary KIM-1 was also significantly increased in early detected inflamed kidney of diabetic patients compared to controls and was positively associated with degree of kidney inflammation [42]. The highest level of KIM1 was found in the DN patients compared to the kidney inflammation state alone, suggesting that urinary KIM-1 excretion could help differentiate kidney inflammation versus DN [42]. In addition, urinary KIM-1 levels were increased in type 2 diabetic patients with normal or mildly increased albuminuria, indicating that tubular and glomerular injuries may coexist at the earliest stage of diabetic kidney disease and KIM-1 could be potential marker of early DN [19].

Urinary KIM-1 excretion is elevated in diabetic patients compared to controls, even before they develop microalbuminuria, indicating that diabetic tubular involvement may precede glomerular involvement and, by measuring KIM-1, this "tubular phase" of renal damage could be detected before albuminuria becomes pathologically elevated $[14,16,18,22$, $32,41]$. Urinary KIM-1 levels seems to predict renal injury secondary to $\mathrm{DN}$ in early period independent of albuminuria, because urinary KIM-1 was elevated despite normal urinary albumin excretion in the normoalbuminuric subgroup [41]. It has been suggested that increased renal biomarkers, such as urinary KIM-1 in diabetics, are early sensitive and specific markers of $\mathrm{DN}$, even preceding the development of microalbuminuria, denoting that they can be used as early and sensitive markers for early detection of DN [18].

In type 2 diabetes, urinary KIM-1 was markedly increased compared with the controls and its levels increased from the normoalbuminuria to the last macroalbuminuria group, predicting the progression of DN [27]. Urinary Kim-1 also was high in normoalbuminuric diabetics before reduction in GFR, indicating early diabetic kidney injury [32]. Furthermore, higher levels of urinary KIM-1 were associated with a faster decline in kidney function and an increased risk of mortality during 4 years of follow-up [40]. Another followup study among type 2 diabetic patients with various degrees of incipient or established DN also found that higher levels of urinary KIM-1 were associated with a faster decline in eGFR [34]. Moreover, low baseline levels of urinary KIM-1 were strongly associated with regression of microalbuminuria independent of clinical characteristics [16]. In another study, Irbesartan treatment significantly reduced levels of the tubular marker urinary KIM-1 in patients with type 2 diabetes and microalbuminuria, indicating the role of KIM-1 in monitoring treatment effect in $\mathrm{DN}$ [43].

\section{Liver-Type Fatty Acid Binding Protein}

Liver-type fatty acid binding protein (L-FABP) is a low molecular weight $(15 \mathrm{kDa})$ intracellular carrier protein that is expressed in the renal proximal tubule and liver. In renal disease L-FABP gene expression in the kidney was upregulated and its urinary excretion was found to correlate with the severity of tubulointerstitial injury, reflecting stresses on the proximal tubules [44]. A study in CKD showed that serum L-FABP levels do not influence the urinary LFABP level, which suggested that the measured L-FABP in urine originates primarily from tubular cells [45]. Urinary L-FABP was significantly higher in diabetic patients compared with healthy controls and correlated with albumin excretion rate and creatinine clearance $(\mathrm{CrCl})$ [46]. Urinary L-FABP levels were significantly higher in diabetic patients with nephropathy than in healthy subjects and correlated positively with urinary albumin, UACR, and albuminuria and inversely with GFR, indicating its possible clinical application as a complementary marker of DN [47-49].

Urinary L-FABP levels were significantly increased in macroalbuminuric patients compared with microalbuminuric and currently normoalbuminuric patients [46]. In one study from type 2 diabetes mellitus patients, elevated levels of urinary L-FABP were evident from the microalbuminuric stage, indicating tubular damage at the early stage of DN [50]. According to this study, urinary excretion of L-FABP levels in the microalbuminuric group was significantly correlated with systolic BP, fasting plasma glucose, and HbAlc, which indicated that urinary L-FABP could be most sensitive marker for detecting glomerular and tubular dysfunction at the early stage of DN [50].

Additionally, urinary levels of L-FABP in patients with microalbuminuria were significantly higher than in those with normoalbuminuria $[18,47,51]$. Increased urinary LFABP excretion has been also found in the micro- and macroalbuminuric patients compared with the patients with 
persistent normoalbuminuria [52]. Urinary L-FABP levels were also elevated in patients with reduced eGFR and showed a positive correlation with systolic $\mathrm{BP}$ and protein/Cr ratio, suggesting the importance of tubular damage in the development of DN and urinary L-FABP excretion in the assessment of tubular dysfunction in early DN [50].

Urinary L-FABP excretion is higher in diabetic patients compared to healthy controls, including in patients without current evidence of nephropathy (normoalbuminuria), indicating its value in detecting $\mathrm{DN}$ even before the appearance of pathological albuminuria, the earlier measurable sign of renal diabetic involvement $[18,48,53,54]$. Urinary levels of L-FABP were significantly higher in the patients with type 2 diabetes who had normoalbuminuria than in normal control subjects and progressively increased in subjects with normo-, micro-, or macroalbuminuria and further increased in patients with ESRD [54]. The levels of urinary L-FABP in each DN group were significantly different from the levels in all of the other groups and significantly increased according to the severity of DN $[47,49,54]$. Levels of urinary LFABP were elevated in normoalbuminuric patients than in the controls and were further increased with increasing levels of albuminuria, indicating its value in accurately reflecting severity of tubular damage in the early stage of DN [48]. In the prospective study, high urinary L-FABP levels were associated with the increase in albuminuria, progression to ESRD, or induction of hemodialysis [54]. Surprisingly, even in the subgroup of patients without renal dysfunction, higher urinary levels of L-FABP were associated with the progression of DN, demonstrating the usefulness of urinary L-FABP as a marker for predicting the progression of $\mathrm{DN}$ in the early stage [54].

Urinary L-FABP was elevated at an early stage, even before any clinical signs of glomerular damage are detectable, and independently predicted the development of microalbuminuria and death, suggesting its value as a useful marker for the detection of tubular damage early in the course of diabetes and for the prediction of DN and death [52]. In addition, urinary concentrations of L-FABP were associated with progress to ESRD in patients with type 2 diabetes, even after adjustment for baseline albuminuria and GFR, indicating the potential importance of urinary L-FABP in identification of persons most likely to progress to ESRD [35]. A long term observational study on type 2 diabetic patients without advanced nephropathy revealed that higher urinary levels of L-FABP were associated with deteriorating renal function and a higher incidence rate of CVD. This association was markedly observed even in patients with normoalbuminuria, which indicated its potential role as a marker for predicting future renal dysfunction and incidence of CVD in diabetic patients with an early stage of nephropathy, independently of albuminuria [51].

\section{N-Acetyl-beta-D-glucosaminidase}

$\mathrm{N}$-acetyl-beta-D-glucosaminidase (NAG) is a hydrolytic lysosomal enzyme found predominantly in proximal tubule. It has been demonstrated as a useful marker of renal tubular impairment in various conditions involved with renal injury or dysfunction [55]. Urinary activities of NAG are elevated in patients with diabetes when compared with nondiabetic control subjects and showed a significant positive correlation with serum Cys C, SCr, UAE, and UACR and an inverse correlation with measured and estimated $\mathrm{CrCl}$ in all patients, indicating the possible clinical application of urinary NAG as a complementary marker for early detection of DN $[14,25$, 56-60]. It is also correlated positively with disease duration and poor glycemic control (HbAlc) [25, 57, 59, 60]. In diabetic patients with poor metabolic control ( $\mathrm{HbAlc}>8 \%$ ), a statistically significant increase in urinary NAG was found compared with the diabetic patients with good metabolic control $[25,60]$.

In diabetics, urine NAG level was significantly higher in microalbuminuric patients compared to both normoalbuminuric patients and controls, suggesting that tubular dysfunction is already present in this period $[16,25,56,57$, 59-61]. Urinary NAG excretion was significantly higher in all patients with type 2 diabetes than in controls and in microalbuminuric than in normoalbuminuric patients, representing early marker of incipient DN [25]. The ROC curve analysis of the above study showed that urinary NAG is the most sensitive marker of microalbuminuria and early renal damage with sensitivity of $83.3 \%$ and specificity of $77.8 \%$ [25].

It was also suggested that NAG had higher sensitivity as urinary marker in early detection of tubular and glomerular lesions in diabetic patients and could be used as screening test for early diagnosis of DN [56]. In another study, urinary NAG and microalbuminuria in the diabetic patients were significantly increased compared to those in the controls and urinary NAG showed the highest sensitivity and specificity (100\% and $87.5 \%$, resp.) as compared to sensitivity and specificity of $\mathrm{SCr}, \mathrm{CrCl}$, and microalbuminuria $(25 \%$ and $24.9 \%, 50 \%$ and $58.3 \%$, and $25 \%$ and $75 \%$, resp.) [60]. The investigators from this study suggested that measuring urinary NAG excretion could be useful for the assessment of renal failure in patients with diabetes and confirmed the use of this enzyme as a routine screening test [60].

Compared to healthy controls, urinary NAG excretion is higher in diabetic patients, even before they develop microalbuminuria $[14,25,56,59,61-64]$. Urinary NAG activity was within the normal ranges in the healthy control groups and significantly increased over the upper reference limit in the groups of patients with normoalbuminuria, indicating the great importance of NAG in discovering the renal tubule cells damage, especially at the early stage before the appearance of microalbuminuria [65]. On the other hand urinary NAG already showed 9-fold increase in the normoalbuminuric patients compared with the controls, whereas albuminuria (and eGFR) in these patients was comparable with controls, which demonstrated the potential value of urinary NAG as sensitive marker of DN as its level increases before other traditional markers become pathologically elevated [14, 62]. Thus, values of the urinary NAG were elevated before microalbuminuria was observed, with the highest values detected in the group of patients with microalbuminuria, indicating that increased excretion of urinary NAG points to early tubular damage and can be used as the most sensitive marker in the early detection of DN $[61,66]$. 
In a more recent study, urinary NAG excretion gradually increases with the increase in duration of diabetes and appeared much before the microalbuminuria, decreased eGFR, and increased SCr. In this study, the urinary NAG activity increased 16- and 18-fold in moderately increased albuminuria and DN patients, respectively, without any change in non-DN patients. A cutoff value of $3 \mathrm{U} / \mathrm{L}$ of urinary NAG has demonstrated a sensitivity of $96.1 \%$ and a specificity of $100 \%$ discriminating healthy controls from patients with microalbuminuria (AUC 0.999) and DN (AUC 1.000), and the investigators concluded that the urinary NAG may be considered as a potential site-specific early tubular damage marker leading to DN [67]. In addition, significantly increased levels of urinary NAG were found in diabetic patients with varying degree of albuminuria and this increase was parallel to the severity of renal involvement expressed with the level of albuminuria [14, 56, 59, 60, 68]. Urinary levels of NAG were higher in patients with diabetes than in controls and increased with increasing categories of albuminuria, suggesting that it could be used as a useful marker reflecting the degree of renal impairment in $\mathrm{DN}[14,59]$.

Urinary NAG activities tended to be higher in diabetic patients with and without albuminuria than in control subjects and differences among the diabetic groups were statistically significant, which implies that this enzyme is a more sensitive marker of tubular damage and could be used as a biomarker for the detection of early stage of DN, even in normoalbuminuric patients. Furthermore, baseline urinary excretion of NAG and rising NAG excretion across time predict both microalbuminuria and macroalbuminuria, which suggested that early NAG excretion may be a marker of susceptibility to DN and combining AER and NAG in repeated measures may help to identify individuals susceptible to DN [69]. Also, low baseline concentrations of urinary NAG were significantly associated with the regression of microalbuminuria over the subsequent 2 years, indicating that tubular dysfunction is a critical component of the early course of DN and urinary NAG can be used as an early marker of normoalbuminuric DN [16].

\section{Alpha-1 Microglobulin}

Alpha-1 microglobulin (A1M) is a small molecular weight protein $(27 \mathrm{kDa})$ present in various body fluids. In the healthy kidney, it passes freely through the glomerular membranes, and about $99 \%$ is reabsorbed and catabolized by the proximal tubular cells. Increased A1M in urine can therefore be an early sign of renal damage, primarily on the proximal tubules [70]. The level of urinary A1M was significantly increased in the group of diabetic patients as compared to the level of normal subjects [71]. Urinary A1M levels were markedly elevated in diabetic patients when compared with control subjects $[61,72-74]$ and correlated directly with urinary albumin excretion, UACR, and serum CysC and negatively with eGFR [72-76], indicating the possible clinical application of urinary $\mathrm{A} 1 \mathrm{M}$ as a complementary marker for early detection of DN. It also correlates with urinary advanced glycation endproducts, diabetes duration, $\mathrm{HbAlc}$, fasting, and postprandial blood glucose $[72,73]$. Urinary A1M level was higher in the patients with poor glucose control (HbAlc $>8.5 \%)$ and directly related to albuminuria [61]. Urinary A1M was also related to the duration, severity, and control of diabetes, indicating that it is a good marker of the severity of renal impairment in type 2 diabetic subjects [75].

A proteomic based study among microalbuminuric diabetic patients showed the early and coappearance of A1M with albumin, demonstrating that urinary A1M can be used as markers for specific and accurate clinical analysis of $\mathrm{DN}$ [77]. The concentration of A1M during the development of albuminuria also showed a very strong positive correlation [61]. In addition, the urinary excretion of A1M was significantly higher in microalbuminuric in comparison with normoalbuminuric patients and controls, indicating tubular damage at an early stage of DN [61, 72, 78]. It also demonstrated the importance of tubular dysfunction, as an early and integral component of the $\mathrm{DN}$ in diabetic patients [73]. A1M is a marker of tubular dysfunction in diabetic patients and hyperglycemia was the most important risk factor associated with A1M urinary excretion, emphasizing the value of tight glycemic control in slowing the progress of tubular dysfunction in diabetic patients $[73,79]$.

In a sample of community treated type 2 diabetic subjects, $45.2 \%$ had elevated A1M urinary excretion, $32.7 \%$ had micro/macroalbuminuria, and $27.2 \%$ had a GFR < $60 \mathrm{~mL} / \mathrm{min}$, indicating tubular dysfunction and nonalbuminuric renal disease in patients with diabetes [79]. In addition to albuminuria measuring glomerular dysfunction, urinary A1M estimating proximal tubular dysfunction is useful for the early detection of nephropathy in diabetes [75]. It was also suggested that urinary A1M provides a noninvasive and inexpensive diagnostic alternative for the early detection of tubular disorders of DN [71].

Urinary A1M is a sensitive biomarker in detecting tubular dysfunction in early DN, even in normoalbuminuric patients, demonstrating that tubular injury is an early event in diabetes [61, 76, 80-83]. Diabetic patients with normoalbuminuria excreted significantly higher levels of urinary A1M compared with healthy individuals, while there was no significant difference between the patients and controls in respect to serum/urine $\mathrm{Cr}$ [61]. In another study, urinary A1M was increased in $27.9 \%$ normoalbuminuric type 2 diabetic patients, indicating that urinary A1M precedes the onset of albuminuria and may serve as a marker in early DN [80]. Elevated levels of urinary A1M in normoalbuminuric patients with diabetes showed that proximal tubule dysfunction may develop before the stage of microalbuminuria and that A1M is a significant biomarker for incipient DN [81]. A1M also correctly identified normoalbuminuric diabetics from healthy controls with accuracy of $89.0 \%$, sensitivity of $86.3 \%$, and specificity of $94.2 \%$ [83]. Urinary A1M levels also significantly increased with severity of albuminuria, indicating its value in predicting progression of $\mathrm{DN}$ as suggested by increasing albuminuria [75].

\section{Beta 2-Microglobulin}

Beta ( $\beta$ ) 2-microglobulin (B2-M) is a low molecular weight protein $(11.8 \mathrm{kDA})$, produced by all cells expressing major 
histocompatibility complex class I antigen. It is readily filtered through the glomerulus and almost completely reabsorbed and catabolized by the renal proximal tubules. Increase in urinary B2-M indicates tubular dysfunction, and measurement of B2-M in urine is a sensitive and reliable assay for detecting tubular injury [84]. The level of B2-M in urine of the patients with diabetes was higher than normal $[85,86]$ and showed significant correlation with urinary albumin excretion and 2-hour postprandial blood sugar [85]. Urine $\mathrm{B} 2-\mathrm{M}$ in the children with type 1 diabetes was significantly increased compared to the controls and correlated positively with disease duration and glycemic control [87].

In type 2 diabetes, significant correlation was found between level of microalbumin and urine B2-M with length of diabetes and serum and urine $\mathrm{Cr}$ [86]. Urinary B2-M excretion is elevated in the patients with a poor metabolic control (HbAlc $>8.0 \%$ ) compared to those with a good one [61]. Urinary B2-M levels were higher in diabetic patients with macro- and/or microvascular complications, indicating that increased urinary $\mathrm{B} 2-\mathrm{M}$ excretion was associated with more severe disease in these patients $[85,88]$. In addition, they appear to be useful in early detection of $\mathrm{DN}$ with positive correlation with the duration of type 1 diabetes and glycemic control (HbAlc) [87]. Most importantly, urine B2-M was able to reliably identify biopsy-proven DN, indicating the potential clinical application of urinary B2-M as a marker for early DN in diabetic patients [89].

Urinary B2-M exhibited a significant positive correlation with urinary albumin levels and negative correlation with GFR and serum B2-M in type 2 DN [90]. This study also found that diabetic kidney damage exhibits an initial increase in urinary B2-M levels, as compared with nondiabetic kidney damage, and renal dysfunction aggravated as urinary B2$M$ levels gradually increased [90]. In addition, urinary B2$M$ excretion is significantly higher in the patients with microalbuminuria than normoalbuminuria and in the controls, indicating the presence of tubular injury in early DN as characterised by increased B2-M excretion [61, 78, 85]. In patients with type 2 diabetes and biopsy-proven $\mathrm{DN}$, urinary excretion of B2-M was significantly correlated with the severity of tubulointerstitial injury, demonstrating the usefulness of B2-M as marker of tubular dysfunction in early DN [91]. A urinary proteomic analysis study found high amounts of B2-M in the urine of diabetic patients with macro- or microalbuminuria compared with controls and patients without micro- or macroalbuminuria [92].

Increased excretion of B2-M was found in early course while albumin excretion was still in normal range in the urine of diabetic patients, which indicated that the increase in urinary B2-M precedes the stage of albuminuria and that early DN is related to proximal tubule dysfunction [80-82]. Urinary B2-M was increased in $23.5 \%$ of normoalbuminuric patients with type 2 diabetes, suggesting that proximal tubule dysfunction may be responsible for early DN independently of preceding glomerular endothelial dysfunction and urinary B2-M may be used as sensitive marker in the diagnosis of early DN [80]. In addition, the urinary excretion of $\mathrm{B} 2-\mathrm{M}$ increased progressively from normoalbuminuria to macroalbuminuria, indicating its value in predicting progression of DN at early stage [85].

\section{Retinol Binding Protein}

Retinol binding protein (RBP) is another low molecular weight protein $(21 \mathrm{kDa})$ which is freely filtered at the glomerulus and then almost completely reabsorbed in the proximal tubule. Both serum and urine levels have been shown to be elevated in patients with diabetes [85, 93, 94]. RBP showed significant positive correlations with triglyceride, systolic BP, and log urinary albumin excretion [93]. Urinary RBP excretion has been found to be increased in diabetic subjects compared with healthy controls [87, 95-97] and to correlate with UAE, serum and urine $\mathrm{Cr}, \mathrm{CrCl}$, and $24 \mathrm{~h}$ urine protein, indicating its potential clinical application as a marker of early DN $[85,95,97]$. It has been also shown to correlate closely with duration of diabetes and glycemic control (HbAlc) [87, 94, 95, 97].

Urinary RBP4 levels were higher in subjects with prediabetes or type 2 diabetes than in subjects with normal glucose tolerance and correlated strongly with fasting glucose, triglycerides, BP, eGFR, and UACR [98]. In addition, urinary excretion of RBP was higher in patients with macroand/or microvascular complications of diabetes compared to those without, which confirmed the utility of RBP as a renal biomarker for predicting diabetic complications $[85,88]$. Urinary RBP was also a predictor of the risk of dialysis, doubling of SCr, or death in diabetic patients with macroalbuminuric $\mathrm{DN}$, suggesting that RBP may serve as a marker to follow-up clinical monitoring of diabetics with DN [99].

Levels of urinary RBP were significantly higher in microalbuminuric diabetics when compared with normoalbuminuric and normal controls, indicating impaired proximal renal tubular function in early stage of $\mathrm{DN}[85,95$, 97]. In one study, diabetic patients with microalbuminuria had concomitant renal tubular disorder indicated by high urinary RBP in $90.9 \%$ of them, which suggested that elevated urinary RBP might be a useful marker of renal injury in early DN [95]. Furthermore, urinary RBP4 was highly predictive of microalbuminuria, even after adjustment for other metabolic parameters. In this study, urinary RBP4 concentration showed a stronger association with urinary ACR than serum RBP4 concentration for microalbuminuria and combined micro- and macroalbuminuria. The AUC (diagnostic accuracy) for urinary RBP4 to detect the presence of microalbuminuria was $0.80 \pm 0.02$ with sensitivity of $80.18 \%$ and specificity of $64.03 \%$; urinary $\mathrm{RBP} 4$ may therefore be used as early stage marker for predicting of diabetic renal damage [98].

Urinary RBP excretion is higher in diabetic patients compared to healthy controls, even before the diagnosis of microalbuminuria $[95,96]$. In the above study, urinary RBP excretion was significantly higher in normoalbuminuric patients than controls and showed a significant correlation with urinary NAG and HbAlc in these patients [95]. Among these normoalbuminuric patients, $82 \%$ had raised urinary excretion of RBP, which suggested that proximal tubular dysfunction may occur independently of glomerular alteration 
[95]. In normoalbuminuric diabetics, the excretion rate of RBP was significantly higher compared to control subjects and correlated to the excretion rate of NAG and albumin [100].

Among type 2 diabetic patients, $50 \%$ were positive for urinary RBP, while $28 \%$ and $6 \%$ of them were positive for micro- and macroalbuminuria, respectively [85]. The increase in the urinary excretion of RBP4 in diabetics is highly specific for tubular disease, which occurs earlier than glomerular (albumin) affection, as urinary RBP4 excretion is increased in early DN and might even be a marker of early renal damage preceding microalbuminuria [96]. Furthermore, the urinary excretion of RBP increased progressively from normoalbuminuria to macroalbuminuria, indicating progression of DN at the early stage [85].

\section{Conclusions}

Tubular injury, as shown by increased urinary tubular damage markers at the microalbuminuria stage of diabetes, is a critical component of the early course of DN. Urinary excretion of these tubular markers is significantly higher in diabetics compared to healthy controls, even before the diagnosis of microalbuminuria, supporting the hypotheses that tubular injury is an early event in diabetes. The tubular markers discriminate between healthy subjects and diabetics in early stages of nephropathy and might also serve as a marker of the efficacy of renal protective agents. Urinary markers of tubular injury are early, sensitive, and specific markers of DN, even preceding the development of microalbuminuria, denoting that they can be used as early and sensitive markers for early detection of DN. Despite the promise of these new tubular injury markers, further large, multicenter prospective studies are still needed to confirm their clinical utility as urinary markers in early $\mathrm{DN}$ for everyday practice.

\section{Abbreviations}

A1M: Alpha- $(\alpha-) 1$ microglobulin

B2-M: $\quad \operatorname{Beta}(\beta)$ 2-microglobulin

DN: Diabetic nephropathy

eGFR: Estimated glomerular filtration rate

KIM-1: Kidney injury molecule-1

L-FABP: Liver-type fatty acid binding protein

NAG: N-Acetyl-beta-glucosaminidase

NGAL: Neutrophil gelatinase associated lipocalin

RBP: Retinol binding protein

UACR: Urinary albumin-creatinine ratio.

\section{Competing Interests}

The authors declare no competing interests.

\section{Acknowledgments}

The authors would like to acknowledge all work leading to this paper.

\section{References}

[1] T. Ninomiya, V. Perkovic, B. E. de Galan et al., "Albuminuria and kidney function independently predict cardiovascular and renal outcomes in diabetes," Journal of the American Society of Nephrology, vol. 20, no. 8, pp. 1813-1821, 2009.

[2] P.-H. Groop, M. C. Thomas, J. L. Moran et al., "The presence and severity of chronic kidney disease predicts all-cause mortality in type 1 diabetes," Diabetes, vol. 58, no. 7, pp. 1651-1658, 2009.

[3] G. Targher, G. Zoppini, M. Chonchol et al., "Glomerular filtration rate, albuminuria and risk of cardiovascular and all-cause mortality in type 2 diabetic individuals," Nutrition, Metabolism and Cardiovascular Diseases, vol. 21, no. 4, pp. 294-301, 2011.

[4] R. C. Atkins and P. Zimmet, "Editorial: Diabetic kidney disease: act now or pay later," Kidney International, vol. 77, no. 5, pp. 375377, 2010.

[5] K. Walczak, M. Sodolska, I. Materek, A. Krysicka, and D. Moczulski, "Impaired renal function in type 2 diabetes patients in the absence of increased urine albumin excretion rate," Diabetologia Doświadczalna i Kliniczna, vol. 8, no. 4, pp. 165$168,2008$.

[6] M. L. Caramori, P. Fioretto, and M. Mauer, "Low glomerular filtration rate in normoalbuminuric type 1 diabetic patients: an indicator of more advanced glomerular lesions," Diabetes, vol. 52, no. 4, pp. 1036-1040, 2003.

[7] P. Budhiraja, B. Thajudeen, and M. Popovtzer, "Absence of albuminuria in type 2 diabetics with classical diabetic nephropathy: clinical pathological study," Journal of Biomedical Science and Engineering, vol. 6, no. 5, pp. 20-25, 2013.

[8] J. Barratt and P. Topham, "Urine proteomics: the present and future of measuring urinary protein components in disease," Canadian Medical Association Journal, vol. 177, no. 4, pp. 361368, 2007.

[9] R. J. MacIsaac and G. Jerums, "Diabetic kidney disease with and without albuminuria," Current Opinion in Nephrology and Hypertension, vol. 20, no. 3, pp. 246-257, 2011.

[10] M. C. Thomas, W. C. Burns, and M. E. Cooper, "Tubular changes in early diabetic nephropathy," Advances in Chronic Kidney Disease, vol. 12, no. 2, pp. 177-186, 2005.

[11] S. C. W. Tang and K. N. Lai, "The pathogenic role of the renal proximal tubular cell in diabetic nephropathy," Nephrology Dialysis Transplantation, vol. 27, no. 8, pp. 3049-3056, 2012.

[12] D. M. Gibb, P. A. Tomlinson, N. R. Dalton, C. Turner, V. Shah, and T. M. Barratt, "Renal tubular proteinuria and microalbuminuria in diabetic patients," Archives of Disease in Childhood, vol. 64, no. 1, pp. 129-134, 1989.

[13] S. C. W. Tang, J. C. K. Leung, and K. N. Lai, "Diabetic tubulopathy: an emerging entity," Diabetes and the Kidney, vol. 170, pp. 124-134, 2011.

[14] F. L. Nauta, W. E. Boertien, S. J. L. Bakker et al., "Glomerular and tubular damage markers are elevated in patients with diabetes," Diabetes Care, vol. 34, no. 4, pp. 975-981, 2011.

[15] J. V. Bonventre, "Can we target tubular damage to prevent renal function decline in diabetes?" Seminars in Nephrology, vol. 32, no. 5, pp. 452-562, 2012.

[16] V. S. Vaidya, M. A. Niewczas, L. H. Ficociello et al., "Regression of microalbuminuria in type 1 diabetes is associated with lower levels of urinary tubular injury biomarkers, kidney injury molecule-1, and N-acetyl- $\beta$-D-glucosaminidase," Kidney International, vol. 79, no. 4, pp. 464-470, 2011. 
[17] A. Lacquaniti, V. Donato, B. Pintaudi et al., "Normoalbuminuric' diabetic nephropathy: tubular damage and NGAL," Acta Diabetologica, vol. 50, no. 6, pp. 935-942, 2013.

[18] S. Abd El Dayem, A. E. El Bohy, and A. El Shehaby, "Value of the intrarenal arterial resistivity indices and different renal biomarkers for early identification of diabetic nephropathy in type 1 diabetic patients," Journal of Pediatric Endocrinology and Metabolism, vol. 29, no. 3, pp. 273-279, 2015.

[19] J. A. de Carvalho, E. Tatsch, B. S. Hausen et al., "Urinary kidney injury molecule-1 and neutrophil gelatinase-associated lipocalin as indicators of tubular damage in normoalbuminuric patients with type 2 diabetes," Clinical Biochemistry, vol. 49, no. 3, pp. 232-236, 2016.

[20] K. Mori and K. Nakao, "Neutrophil gelatinase-associated lipocalin as the real-time indicator of active kidney damage," Kidney International, vol. 71, no. 10, pp. 967-970, 2007.

[21] S. S. Kim, S. H. Song, I. J. Kim et al., "Clinical implication of urinary tubular markers in the early stage of nephropathy with type 2 diabetic patients," Diabetes Research and Clinical Practice, vol. 97, no. 2, pp. 251-257, 2012.

[22] S. E. Nielsen, K. J. Schjoedt, A. S. Astrup et al., "Neutrophil gelatinase-associated lipocalin (NGAL) and kidney injury molecule 1 (KIM1) in patients with diabetic nephropathy: a cross-sectional study and the effects of lisinopril," Diabetic Medicine, vol. 27, no. 10, pp. 1144-1150, 2010.

[23] M. H. Hafez, F. A. F. El-Mougy, S. H. Makar, and S. S. Abd El Shaheed, "Detection of an earlier tubulopathy in diabetic nephropathy among children with normoalbuminuria," Iranian Journal of Kidney Diseases, vol. 9, no. 2, pp. 126-131, 2015.

[24] Z. Yürük Yıldırım, A. Nayır, A. Yılmaz, A. Gedikbaşı, and R. Bundak, "Neutrophil gelatinase-associated lipocalin as an early sign of diabetic kidney injury in children," Journal of Clinical Research in Pediatric Endocrinology, vol. 7, no. 4, pp. 274-279, 2015.

[25] H. S. Assal, S. Tawfeek, E. A. Rasheed, D. El-Lebedy, and E. $\mathrm{H}$. Thabet, "Serum cystatin $\mathrm{C}$ and tubular urinary enzymes as biomarkers of renal dysfunction in type 2 diabetes mellitus," Clinical Medicine Insights: Endocrinology and Diabetes, vol. 6, pp. 7-13, 2013.

[26] D. Bolignano, A. Lacquaniti, G. Coppolino et al., "Neutrophil gelatinase-associated lipocalin as an early biomarker of nephropathy in diabetic patients," Kidney and Blood Pressure Research, vol. 32, no. 2, pp. 91-98, 2009.

[27] W.-J. Fu, S.-L. Xiong, Y.-G. Fang et al., "Urinary tubular biomarkers in short-term type 2 diabetes mellitus patients: a crosssectional study," Endocrine, vol. 41, no. 1, pp. 82-88, 2012.

[28] J. Zachwieja, J. Soltysiak, P. Fichna et al., "Normal-range albuminuria does not exclude nephropathy in diabetic children," Pediatric Nephrology, vol. 25, no. 8, pp. 1445-1451, 2010.

[29] A. A. Al-Refai, S. I. Tayel, A. Ragheb, A. G. Dala, and A. Zahran, "Urinary neutrophil gelatinase associated lipocalin as a marker of tubular damage in type 2 diabetic patients with and without albuminuria," Open Journal of Nephrology, vol. 4, no. 1, pp. 3746, 2014.

[30] V. Garg, M. Kumar, H. S. Mahapatra, A. Chitkara, A. K. Gadpayle, and V. Sekhar, "Novel urinary biomarkers in pre-diabetic nephropathy," Clinical and Experimental Nephrology, vol. 19, no. 5, pp. 895-900, 2015.

[31] K. Demir, A. Abaci, T. Küme, A. Altincik, G. Çatli, and E. Böber, "Evaluation of neutrophil gelatinase-associated lipocalin in normoalbuminuric normotensive type 1 diabetic adolescents,"
Journal of Pediatric Endocrinology and Metabolism, vol. 25, no. 5-6, pp. 517-523, 2012.

[32] A. Ucakturk, B. Avci, G. Genc, O. Ozkaya, and M. Aydin, "Kidney injury molecule-1 and neutrophil gelatinase associated lipocalin in normoalbuminuric diabetic children," Journal of Pediatric Endocrinology and Metabolism, vol. 29, no. 2, pp. 145151, 2015.

[33] J. Wu, Y. Ding, C. Zhu et al., "Urinary TNF- $\alpha$ and NGAL are correlated with the progression of nephropathy in patients with type 2 diabetes," Experimental and Therapeutic Medicine, vol. 6, no. 6, pp. 1482-1488, 2013.

[34] S. E. Nielsen, H. Reinhard, D. Zdunek et al., "Tubular markers are associated with decline in kidney function in proteinuric type 2 diabetic patients," Diabetes Research and Clinical Practice, vol. 97, no. 1, pp. 71-76, 2012.

[35] G. D. Fufaa, E. J. Weil, R. G. Nelson et al., "Association of urinary KIM-1, L-FABP, NAG and NGAL with incident end-stage renal disease and mortality in American Indians with type 2 diabetes mellitus," Diabetologia, vol. 58, no. 1, pp. 188-198, 2015.

[36] J. V. Bonventre, "Kidney injury molecule-1 (KIM-1): a urinary biomarker and much more," Nephrology Dialysis Transplantation, vol. 24, no. 11, pp. 3265-3268, 2009.

[37] W. K. Han, V. Bailly, R. Abichandani, R. Thadhani, and J. V. Bonventre, "Kidney Injury Molecule-1 (KIM-1): a novel biomarker for human renal proximal tubule injury," Kidney International, vol. 62, no. 1, pp. 237-244, 2002.

[38] A. C. Carlsson, M. Calamia, U. Risérus et al., "Kidney injury molecule (KIM)-1 is associated with insulin resistance: results from two community-based studies of elderly individuals," Diabetes Research and Clinical Practice, vol. 103, no. 3, pp. 516521, 2014.

[39] N. E. El-Ashmawy, E. A. El-Zamarany, N. F. Khedr, A. I. Abd ElFattah, and S. A. Eltoukhy, "Kidney injury molecule-1 (Kim-1): an early biomarker for nephropathy in type II diabetic patients," International Journal of Diabetes in Developing Countries, vol. 35, no. S3, pp. 431-438, 2015.

[40] B. R. Conway, D. Manoharan, D. Manoharan et al., "Measuring urinary tubular biomarkers in type 2 diabetes does not add prognostic value beyond established risk factors," Kidney International, vol. 82, no. 7, pp. 812-818, 2012.

[41] B. K. Tekce, H. Tekce, G. Aktas, and M. Sit, "Evaluation of the urinary kidney injury molecule-1 levels in patients with diabetic nephropathy," Clinical and Investigative Medicine, vol. 37, no. 6, pp. E377-E383, 2014.

[42] S. A. Ahmed and M. A. Hamed, "Kidney injury molecule-1 as a predicting factor for inflamed kidney, diabetic and diabetic nephropathy Egyptian patients," Journal of Diabetes and Metabolic Disorders, vol. 14, no. 1, article 6, 2015.

[43] S. E. Nielsen, K. Rossing, G. Hess et al., "The effect of RAAS blockade on markers of renal tubular damage in diabetic nephropathy: U-NGAL, u-KIM1 and u-LFABP," Scandinavian Journal of Clinical and Laboratory Investigation, vol. 72, no. 2, pp. 137-142, 2012.

[44] A. Kamijo, T. Sugaya, A. Hikawa et al., "Urinary excretion of fatty acid-binding protein reflects stress overload on the proximal tubules," American Journal of Pathology, vol. 165, no. 4, pp. 1243-1255, 2004.

[45] A. Kamijo, T. Sugaya, A. Hikawa et al., "Urinary liver-type fatty acid binding protein as a useful biomarker in chronic kidney disease," Molecular and Cellular Biochemistry, vol. 284, no. 1-2, pp. 175-182, 2006. 
[46] M. Von Eynatten, M. Baumann, U. Heemann et al., "Urinary LFABP and anaemia: distinct roles of urinary markers in type 2 diabetes," European Journal of Clinical Investigation, vol. 40, no. 2, pp. 95-102, 2010.

[47] T. Nakamura, T. Sugaya, Y. Kawagoe, Y. Ueda, S. Osada, and H. Koide, "Effect of pitavastatin on urinary liver-type fatty acid-binding protein levels in patients with early diabetic nephropathy," Diabetes Care, vol. 28, no. 11, pp. 2728-2732, 2005.

[48] S. E. Nielsen, T. Sugaya, L. Tarnow et al., "Tubular and glomerular injury in diabetes and the impact of ACE inhibition," Diabetes Care, vol. 32, no. 9, pp. 1684-1688, 2009.

[49] K. Suzuki, T. Babazono, H. Murata, and Y. Iwamoto, "Clinical significance of urinary liver-type fatty acid-binding protein in patients with diabetic nephropathy," Diabetes Care, vol. 28, no. 8, pp. 2038-2039, 2005.

[50] V. Viswanathan, S. Sivakumar, V. Sekar, D. Umapathy, and S. Kumpatla, "Clinical significance of urinary liver-type fatty acid binding protein at various stages of nephropathy," Indian Journal of Nephrology, vol. 25, no. 5, pp. 269-273, 2015.

[51] S.-I. Araki, M. Haneda, D. Koya et al., "Predictive effects of urinary liver-type fatty acid-binding protein for deteriorating renal function and incidence of cardiovascular disease in type 2 diabetic patients without advanced nephropathy," Diabetes Care, vol. 36, no. 5, pp. 1248-1253, 2013.

[52] S. E. Nielsen, T. Sugaya, P. Hovind, T. Baba, H.-H. Parving, and P. Rossing, "Urinary liver-type fatty acid-binding protein predicts progression to nephropathy in type 1 diabetic patients," Diabetes Care, vol. 33, no. 6, pp. 1320-1324, 2010.

[53] N. M. Panduru, C. Forsblom, M. Saraheimo et al., "Urinary liver-type fatty acid-binding protein and progression of diabetic nephropathy in type 1 diabetes," Diabetes Care, vol. 36, no. 7, pp. 2077-2083, 2013.

[54] A. Kamijo-Ikemori, T. Sugaya, T. Yasuda et al., "Clinical significance of urinary liver-type fatty acid-binding protein in diabetic nephropathy of type 2 diabetic patients," Diabetes Care, vol. 34 , no. 3, pp. 691-696, 2011.

[55] S. Skálová, “The diagnostic role of urinary N-acetyl-beta-Dglucosaminidase (NAG) activity in the detection of renal tubular impairment," Acta Medica, vol. 48, no. 2, pp. 75-80, 2005.

[56] S. Uslu, B. Efe, Ö. Alataş et al., "Serum cystatin C and urinary enzymes as screening markers of renal dysfunction in diabetic patients," Journal of Nephrology, vol. 18, no. 5, pp. 559-567, 2005.

[57] B. R. Bouvet, C. V. Paparella, S. M. M. Arriaga, A. L. Monje, A. M. Amarilla, and A. M. Almará, "Evaluation of urinary $\mathrm{N}$-acetyl-beta-D-glucosaminidase as a marker of early renal damage in patients with type 2 diabetes mellitus," Arquivos Brasileiros de Endocrinologia e Metabologia, vol. 58, no. 8, pp. 798-801, 2014.

[58] F. P. Udomah, U. E. Ekrikpo, E. Effa, B. Salako, A. Arije, and S. Kadiri, "Association between urinary N-acetyl-betaD-glucosaminidase and microalbuminuria in diabetic black Africans," International Journal of Nephrology, vol. 2012, Article ID 235234, 5 pages, 2012.

[59] G. Sheira, N. Noreldin, A. Tamer, and M. Saad, "Urinary biomarker $\mathrm{N}$-acetyl- $\beta$-D-glucosaminidase can predict severity of renal damage in diabetic nephropathy," Journal of Diabetes and Metabolic Disorders, vol. 14, article 4, 2015.

[60] A. Mohammadi-Karakani, S. Asgharzadeh-Haghighi, M. Ghazi-Khansari, and R. Hosseini, "Determination of urinary enzymes as a marker of early renal damage in diabetic patients,"
Journal of Clinical Laboratory Analysis, vol. 21, no. 6, pp. 413-417, 2007.

[61] G. Nikolov, M. Boncheva, T. Gruev, S. Biljali, O. StojcevaTaneva, and E. Masim-Spasovska, "Urinary biomarkers in the early diagnosis of renal damage in diabetes mellitus patients," Scripta Scientifica Medica, vol. 45, no. 3, pp. 58-64, 2013.

[62] W. K. Gatua, J. N. Makumi, E. M. Njagi, C. S. Kigondu, S. O. Mcligeyo, and S. K. Waithaka, "Evaluation of urinary tubular enzymes as screening markers of renal dysfunction in patients suffering from diabetes mellitus," Asian Journal of Medical Sciences, vol. 3, no. 3, pp. 84-90, 2011.

[63] R. Bansal, D. Lahon, B. B. Thakur, and A. Saikia, "A study of urinary enzymes as a marker of early renal damage in patients suffering from diabetes mellitus," Indian Journal of Basic \& Applied Medical Research, vol. 4, no. 4, pp. 768-776, 2015.

[64] V. Ambade, P. Singh, B. L. Somani, and D. Basannar, "Urinary $\mathrm{N}$-acetyl beta glucosaminidase and gamma glutamyl transferase as early markers of diabetic nephropathy," Indian Journal of Clinical Biochemistry, vol. 21, no. 2, pp. 142-148, 2006.

[65] V. Vlatković, B. Stojimirović, and R. Obrenović, "Damage of tubule cells in diabetic nephropathy type 2 : urinary $\mathrm{N}$-acetyl- $\beta$ D-glucosaminidasis and $\gamma$-glutamil-transferasis," Vojnosanitetski Pregled, vol. 64, no. 2, pp. 123-127, 2007.

[66] V. Vlatković, B. Stojimirović, R. Obrenović, and S. Nogić, "Damage to proximal tubular epithelial cells in type 2 diabetes mellitus," Medicinski pregled, vol. 60, no. 5-6, pp. 272-276, 2007.

[67] D. N. Patel and K. Kalia, "Efficacy of urinary N-acetyl- $\beta$-Dglucosaminidase to evaluate early renal tubular damage as a consequence of type 2 diabetes mellitus: a cross-sectional study," International Journal of Diabetes in Developing Countries, vol. 35, no. 3, supplement, pp. 449-457, 2015.

[68] A. Piwowar, M. Knapik-Kordecka, I. Fus, and M. Warwas, "Urinary activities of cathepsin B, N-acetyl- $\beta$-D-glucosaminidase, and albuminuria in patients with type 2 diabetes mellitus," Medical Science Monitor, vol. 12, no. 5, pp. CR210-CR214, 2006.

[69] E. F. O. Kern, P. Erhard, W. Sun, S. Genuth, and M. F. Weiss, "Early urinary markers of diabetic kidney disease: a nested casecontrol study from the diabetes Control and Complications Trial (DCCT)," American Journal of Kidney Diseases, vol. 55, no. 5, pp. 824-834, 2010.

[70] O. Bakoush, A. Grubb, B. Rippe, and J. Tencer, "Urine excretion of protein $\mathrm{HC}$ in proteinuric glomerular diseases correlates to urine IgG but not to albuminuria," Kidney International, vol. 60, no. 5, pp. 1904-1909, 2001.

[71] N. Shore, R. Khurshid, and M. Saleem, "Alpha-1 microglobulin: a marker for early detection of tubular disorders in diabetic nephropathy," Journal of Ayub Medical College, vol. 22, no. 4, pp. 53-55, 2010.

[72] L. Petrica, A. Vlad, G. Gluhovschi et al., "Glycated peptides are associated with proximal tubule dysfunction in type 2 diabetes mellitus," International Journal of Clinical and Experimental Medicine, vol. 8, no. 2, pp. 2516-2525, 2015.

[73] A. Saif and N. Soliman, "Urinary $\alpha_{1}$-microglobulin and albumin excretion in children and adolescents with type 1 diabetes," Journal of Diabetes, 2016.

[74] E. Korpinen, A.-M. Teppo, L. Hukkanen, H. K. Åkerblom, C. Grönhagen-Riska, and O. Vaarala, "Urinary transforming growth factor- $\beta 1$ and $\alpha 1$-microglobulin in children and adolescents with type 1 diabetes," Diabetes Care, vol. 23, no. 5, pp. 664$668,2000$.

[75] C.-Y. Hong, K. Hughes, K.-S. Chia, V. Ng, and S.-L. Ling, "Urinary $\alpha 1$-microglobulin as a marker of nephropathy in type 
2 diabetic Asian subjects in Singapore," Diabetes Care, vol. 26, no. 2, pp. 338-342, 2003.

[76] L. Petrica, A. Vlad, G. Gluhovschi et al., "Proximal tubule dysfunction is associated with podocyte damage biomarkers nephrin and vascular endothelial growth factor in type 2 diabetes mellitus patients: a cross-sectional study," PLOS ONE, vol. 9, no. 11, Article ID el12538, 2014.

[77] S. Jain, A. Rajput, Y. Kumar, N. Uppuluri, A. S. Arvind, and U. Tatu, "Proteomic analysis of urinary protein markers for accurate prediction of diabetic kidney disorder," Journal of Association of Physicians of India, vol. 53, pp. 513-520, 2005.

[78] A. Kalansooriya, I. Holbrook, P. Jennings, and P. H. Whiting, "Serum cystatin C, enzymuria, tubular proteinuria and early renal insult in type 2 diabetes," British Journal of Biomedical Science, vol. 64, no. 3, pp. 121-123, 2007.

[79] M. L. Robles-Osorio and E. Sabath, "Tubular dysfunction and non-albuminuric renal disease in subjects with type 2 diabetes mellitus," Revista de Investigacion Clinica, vol. 66, no. 3, pp. 234239, 2014.

[80] L. Petrica, M. Petrica, A. Vlad et al., "Proximal tubule dysfunction is dissociated from endothelial dysfunction in normoalbuminuric patients with type 2 diabetes mellitus: a cross-sectional study," Nephron Clinical Practice, vol. 118, no. 2, pp. c155-c164, 2011.

[81] L. Petrica, M. Petrica, A. Vlad et al., "Nephro- and neuroprotective effects of rosiglitazone versus glimepiride in normoalbuminuric patients with type 2 diabetes mellitus: a randomized controlled trial," Wiener Klinische Wochenschrift, vol. 121, no. 2324, pp. 765-775, 2009.

[82] L. Petrica, A. Vlad, M. Petrica et al., "Pioglitazone delays proximal tubule dysfunction and improves cerebral vessel endothelial dysfunction in normoalbuminuric people with type 2 diabetes mellitus," Diabetes Research and Clinical Practice, vol. 94, no. 1, pp. 22-32, 2011.

[83] D. A. Brott, S. T. Furlong, S. H. Adler et al., "Characterization of renal biomarkers for use in clinical trials: effect of preanalytical processing and qualification using samples from subjects with diabetes," Drug Design, Development and Therapy, vol. 9, pp. 3191-3198, 2015.

[84] X. Zeng, D. Hossain, D. G. Bostwick, G. A. Herrera, B. Ballester, and P. L. Zhang, "Urinary $\beta 2$-microglobulin is a sensitive indicator for renal tubular injury," Scholarena Journal (SAJ) of Case Reports, vol. 1, no. 1, pp. 1-6, 2014.

[85] C. Y. Hong, K. S. Chia, and S. L. Ling, "Urine protein excretion among Chinese patients with type 2 diabetes mellitus," The Medical Journal of Malaysia, vol. 55, no. 2, pp. 220-229, 2000.

[86] N. Aghamohammadzadeh, A. A. Abolfathi, A. Alizadeh, and H. Ghasemi, "Evaluation of microalbuminuria and urine beta 2 microglobulin in patients with type 1 and 2 diabetes," International Journal of Current Research and Academic Review, vol. 3 , no. 6, pp. 451-462, 2015.

[87] M. A. Fathy, M. M. Elkady, H. A. Fathy, S. A. Awad, and A. A. Elmenshawy, "Estimation of renal tubular markers for predicting early stage diabetic nephropathy in Egyptian children with type I dibetes mellitus," Research Journal of Medical Sciences, vol. 4, no. 2, pp. 207-211, 2009.

[88] C. Y. Hong, K. S. Chia, and S. L. Ling, "Urinary protein excretion in Type 2 diabetes with complications," Journal of Diabetes and its Complications, vol. 14, no. 5, pp. 259-265, 2000.

[89] M. Papale, S. Di Paolo, R. Magistroni et al., "Urine proteome analysis may allow noninvasive differential diagnosis of diabetic nephropathy," Diabetes Care, vol. 33, no. 11, pp. 2409-2415, 2010.
[90] X. Rao, M. Wan, C. Qiu, and C. Jiang, "Role of cystatin C in renal damage and the optimum cut-off point of renal damage among patients with type 2 diabetes mellitus," Experimental and Therapeutic Medicine, vol. 8, no. 3, pp. 887-892, 2014.

[91] K. Mise, J. Hoshino, T. Ueno et al., "Prognostic value of tubulointerstitial lesions, urinary $\mathrm{N}$-acetyl- $\beta$-D-glucosaminidase, and urinary $\beta 2$-microglobulin in patients with type 2 diabetes and biopsy-proven diabetic nephropathy," Clinical Journal of the American Society of Nephrology, vol. 11, no. 4, pp. 593-601, 2016.

[92] H. Dihazi, G. A. Müller, S. Lindner et al., "Characterization of diabetic nephropathy by urinary proteomic analysis: identification of a processed ubiquitin form as a differentially excreted protein in diabetic nephropathy patients," Clinical Chemistry, vol. 53, no. 9, pp. 1636-1645, 2007.

[93] K. Takebayashi, M. Suetsugu, S. Wakabayashi, Y. Aso, and T. Inukai, "Retinol binding protein-4 levels and clinical features of type 2 diabetes patients," Journal of Clinical Endocrinology and Metabolism, vol. 92, no. 7, pp. 2712-2719, 2007.

[94] E. Novery, S. Susanah, and D. Rachmadi, "The correlation of urine retinol binding protein-4 and serum HbAlc with glomerular filtration rate in type 1 (insulin-dependent) diabetic children: a perspective on the duration of diabetes," Open Journal of Pediatrics, vol. 5, no. 2, pp. 134-140, 2015.

[95] M. A. K. Salem, S. A. El-Habashy, O. M. Saeid, M. M. K. El-Tawil, and P. H. Tawfik, "Urinary excretion of $n$-acetyl- $\beta$ D-glucosaminidase and retinol binding protein as alternative indicators of nephropathy in patients with type 1 diabetes mellitus," Pediatric Diabetes, vol. 3, no. 1, pp. 37-41, 2002.

[96] A. A. S. Abdallah, K. A. El-Shamy, W. M. Morcos, T. H. Mikhail, and N. N. Fadl, "Early marker for renal impairment and angiopathy in diabetic Egyptian children," Life Science Journal, vol. 8, no. 3, pp. 358-366, 2011.

[97] N. Zahra, M. A. Javad, and N. Aboalfazl, "Detection of early stage renal disease by elevation of certain low molecular Weight proteins in urine of diabetes patients," International Journal of Biological Sciences and Applications, vol. 1, no. 1, pp. 15-18, 2014.

[98] S. E. Park, N. S. Lee, J. W. Park et al., "Association of urinary RBP4 with insulin resistance, inflammation, and microalbuminuria," European Journal of Endocrinology, vol. 171, no. 4, pp. 443-449, 2014.

[99] S. M. Titan, J. M. Vieira Jr., W. V. Dominguez et al., "Urinary MCP-1 and RBP: independent predictors of renal outcome in macroalbuminuric diabetic nephropathy," Journal of Diabetes and its Complications, vol. 26, no. 6, pp. 546-553, 2012.

[100] C. Catalano, P. H. Winocour, S. Parlongo, I. Gibb, S. Gillespie, and K. G. M. M. Alberti, "Measures of tubular function in normoalbuminuric insulin-dependent diabetic patients and their relationship with sodium lithium countertransport activity," Nephron, vol. 73, no. 4, pp. 613-618, 1996. 


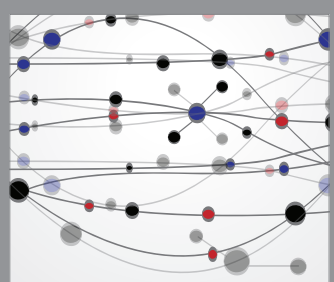

The Scientific World Journal
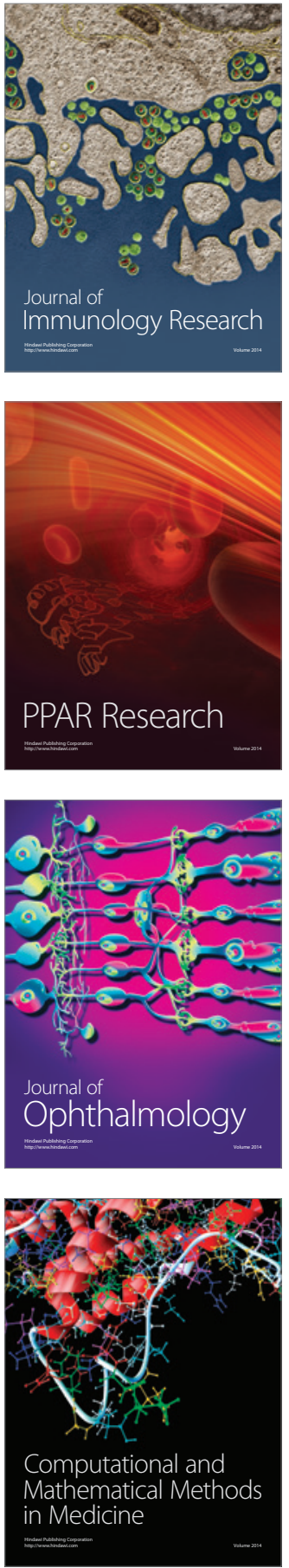

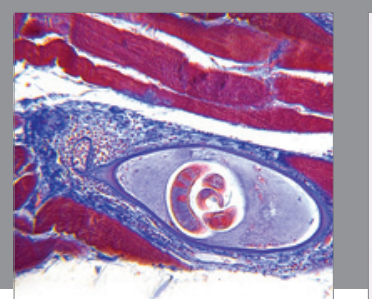

Gastroenterology Research and Practice

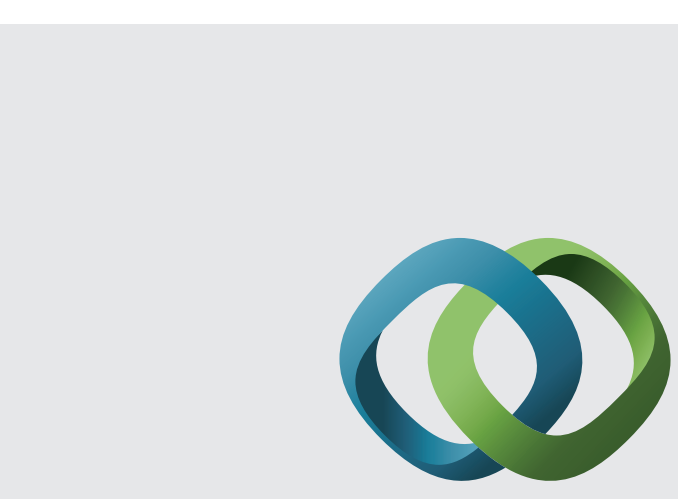

\section{Hindawi}

Submit your manuscripts at

http://www.hindawi.com
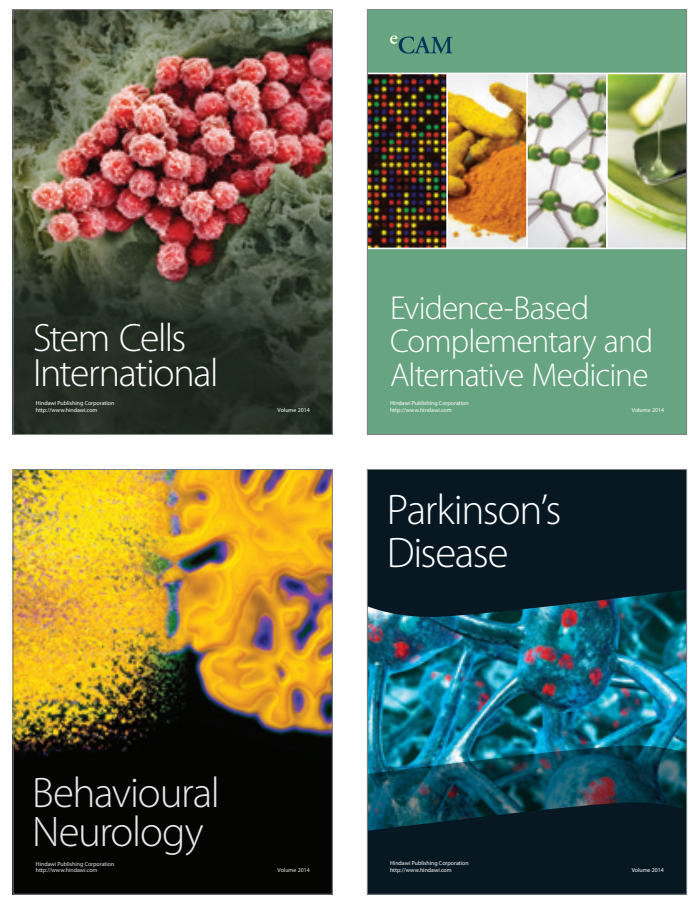
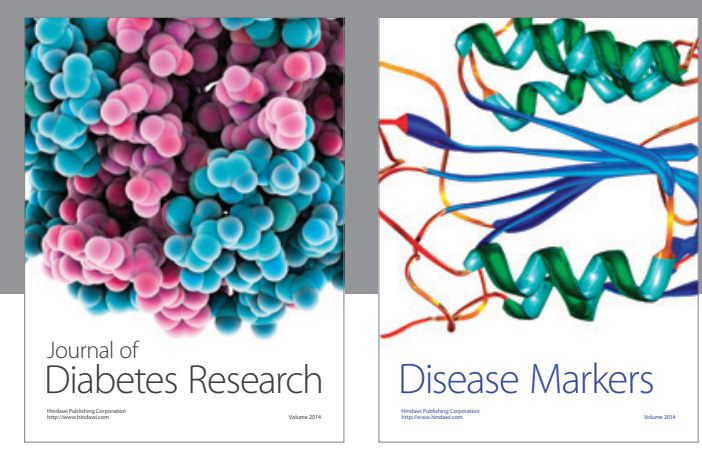

Disease Markers
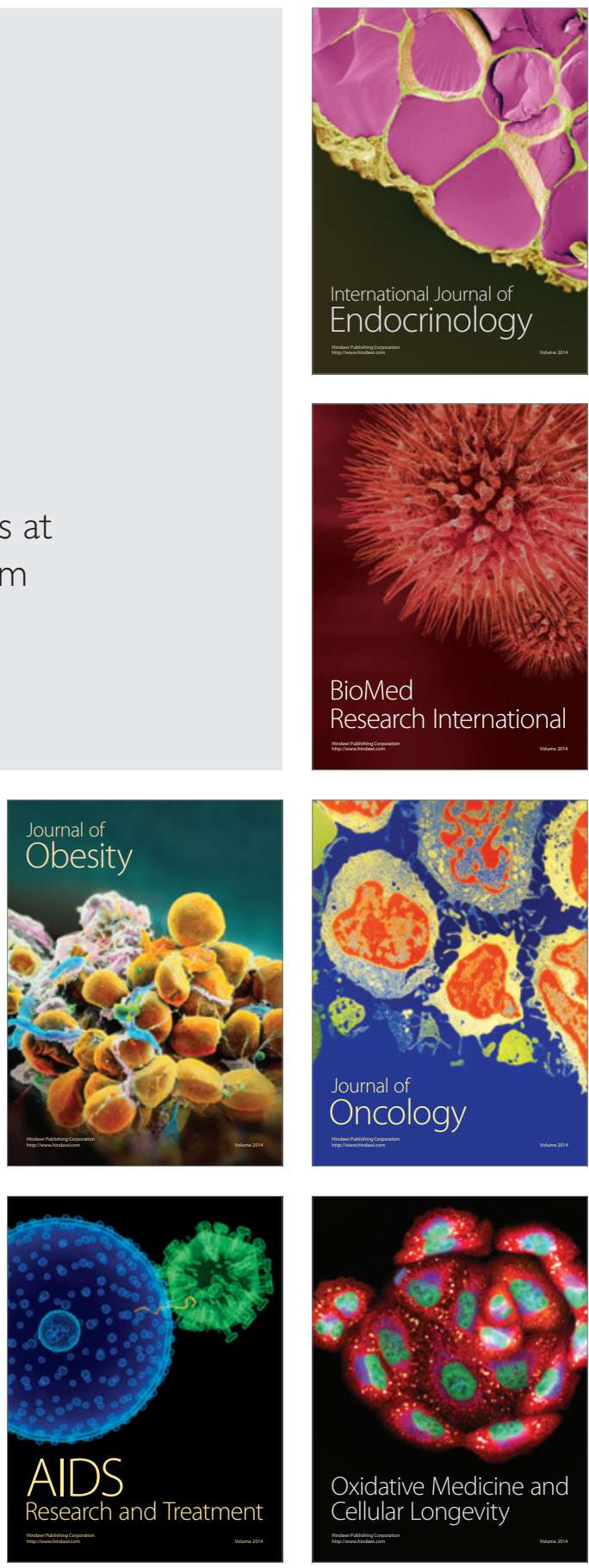